\title{
Mechanical Power during Veno-Venous Extracorporeal Membrane Oxygenation Initiation: A Pilot-Study
}

\author{
Mirko Belliato $^{1} \mathbb{D}$, Francesco Epis ${ }^{1, * \mathbb{D}}$, Luca Cremascoli ${ }^{2} \mathbb{D}$, Fiorenza Ferrari ${ }^{3,4} \mathbb{D}$, Maria Giovanna ,uattrone $^{2}$, \\ Christoph Fisser ${ }^{5}$ (D), Maximilian Valentin Malfertheiner ${ }^{5}$, Fabio Silvio Taccone ${ }^{6}$, Matteo Di Nardo ${ }^{7}$ (D), \\ Lars Mikael Broman ${ }^{8}$ (D) and Roberto Lorusso 9,10
}

\section{check for}

updates

Citation: Belliato, M.; Epis, F.; Cremascoli, L.; Ferrari, F.; Quattrone, M.G.; Fisser, C.; Malfertheiner, M.V.; Taccone, F.S.; Di Nardo, M.; Broman, L.M.; et al. Mechanical Power during Veno-Venous Extracorporeal Membrane Oxygenation Initiation: A Pilot-Study. Membranes 2021, 11, 30. https://doi.org/10.3390/membranes 11010030

Received: 18 November 2020 Accepted: 29 December 2020 Published: 2 January 2021

Publisher's Note: MDPI stays neutral with regard to jurisdictional clai$\mathrm{ms}$ in published maps and institutional affiliations.

Copyright: $\odot 2021$ by the authors. Licensee MDPI, Basel, Switzerland. This article is an open access article distributed under the terms and conditions of the Creative Commons Attribution (CC BY) license (https:// creativecommons.org/licenses/by/ $4.0 /)$.
1 2nd Intensive Care Unit, UOC Anestesia e Rianimazione II Cardiopolmonare, Fondazione IRCCS Policlinico San Matteo, 27100 Pavia, Italy; m.belliato@gmail.com

2 Department of Clinical-Surgical, Diagnostic and Paediatric Sciences, Unit of Anaesthesia and Intensive Care, University of Pavia, 27100 Pavia, Italy; doc.cremascoli@gmail.com (L.C.); magiqu@gmail.com (M.G.Q.)

3 1st Intensive Care Unit, UOC Anestesia e Rianimazione I, Fondazione IRCCS Policlinico San Matteo, 27100 Pavia, Italy; fioreferrari28@gmail.com

4 International Renal Research Institute of Vicenza (IRRIV) and Department of Nephrology, Dialysis and Transplantation, 36100 Vicenza, Italy

5 Department of Internal Medicine II, Cardiology and Pneumology, Intensive Care, University Hospital Regensburg, 93053 Regensburg, Germany; christoph.fisser@ukr.de (C.F.); maxmalfertheiner@gmail.com (M.V.M.)

6 Department of Intensive Care, Erasme Hospital, Université Libre de Bruxelles, Cliniques Universitaires de Brussels, 1070 Brussels, Belgium; ftaccone@ulb.ac.be

7 Pediatric Intensive Care, Bambino Gesù Children's Hospital, IRCCS, 00165 Rome, Italy; matteo.dinardo@opbg.net

8 ECMO Centre Karolinska, Astrid Lindgren Children's Hospital, Karolinska University Hospital, and Department of Physiology and Pharmacology, Karolinska Institutet, 17164 Solna (Stockholm), Sweden; lars.broman@sll.se

9 Department of Cardio-Thoracic Surgery, Heart and Vascular Centre, Maastricht University Medical Centre (MUMC), 6229 HX Maastricht, The Netherlands; roberto.lorussobs@gmail.com

10 Cardiovascular Research Institute Maastricht (CARIM), 6229 ER Maastricht, The Netherlands

* Correspondence: francesco.epis01@gmail.com; Tel.: +39-0382-503524

Abstract: Mechanical power (MP) represents a useful parameter to describe and quantify the forces applied to the lungs during mechanical ventilation (MV). In this multi-center, prospective, observational study, we analyzed MP variations following MV adjustments after veno-venous extra-corporeal membrane oxygenation (VV ECMO) initiation. We also investigated whether the MV parameters (including MP) in the early phases of VV ECMO run may be related to the intensive care unit (ICU) mortality. Thirty-five patients with severe acute respiratory distress syndrome were prospectively enrolled and analyzed. After VV ECMO initiation, we observed a significant decrease in median MP (32.4 vs. $8.2 \mathrm{~J} / \mathrm{min}, p<0.001$ ), plateau pressure ( 27 vs. $21 \mathrm{cmH}_{2} \mathrm{O}, p=0.012$ ), driving pressure (11 vs. $\left.8 \mathrm{cmH}_{2} \mathrm{O}, p=0.014\right)$, respiratory rate ( $\mathrm{RR}, 22$ vs. 14 breaths $\left./ \mathrm{min}, p<0.001\right)$, and tidal volume adjusted to patient ideal body weight $\left(\mathrm{V}_{\mathrm{T}} / \mathrm{IBW}, 5.5 \mathrm{vs.} 4.0 \mathrm{~mL} / \mathrm{kg}, p=0.001\right)$ values. During the early phase of ECMO run, RR (17 vs. 13 breaths $/ \mathrm{min}, p=0.003$ ) was significantly higher, while positive end-expiratory pressure (10 vs. $\left.14 \mathrm{cmH}_{2} \mathrm{O}, p=0.048\right)$ and $\mathrm{V}_{\mathrm{T}} / \mathrm{IBW}(3.0 \mathrm{vs} .4 .0 \mathrm{~mL} / \mathrm{kg}, p=0.028)$ were lower in ICU non-survivors, when compared to the survivors. The observed decrease in MP after ECMO initiation did not influence ICU outcome. Waiting for large studies assessing the role of these parameters in VV ECMO patients, RR and MP monitoring should not be underrated during ECMO.

Keywords: veno-venous extracorporeal membrane oxygenation; acute respiratory distress syndrome; ventilator-induced lung injury; mechanical ventilation; mechanical power; respiratory rate

\section{Introduction}

The complex interaction between mechanical ventilation (MV) and the native lung may promote ventilator-induced lung injury (VILI), especially in patients suffering from 
the acute respiratory distress syndrome (ARDS) [1], which would lead to gas exchange impairment and decreased respiratory system compliance [2]. The concept of lung protective ventilation has been developed and proven to reduce mortality in ARDS patients [3]. Low tidal volume $\left(\mathrm{V}_{\mathrm{T}}\right)$, low driving pressure $(\Delta \mathrm{P})[4,5]$, and high positive end-expiratory pressure (PEEP) are recommended to minimize the forces applied to the lungs and to avoid cyclic collapse and reopening of alveoli [6].

Nowadays, extracorporeal membrane oxygenation (ECMO) has become an effective and safe intervention in severe ARDS, with an increasing use in clinical practice $[7,8]$. In the absence of any standardized protocol, strategies to mitigate VILI during ECMO [9] rely on expert opinions [10]; nevertheless, it is generally accepted that reduced VILI would enhance lung recovery also in these patients [11,12].

To perform lung protective ventilation, it is necessary to understand how to reduce the forces applied by MV on lung tissue [13]. Stress and strain are difficult to measure in daily practice. As such, an available parameter which accounts for most of the potential causes of VILI has been recently introduced [14,15]: the so-called "mechanical power" (MP) represents the total energy delivered within a given time frame to the respiratory system, expressed in joules/minute $(\mathrm{J} / \mathrm{min})$ [16]. MP is the sum of the forces acting on the lung surface during $\mathrm{MV}$, which are, according to the equation of motion: respiratory rate $(\mathrm{RR}), \mathrm{V}_{\mathrm{T}}$, respiratory system elastance, inspiratory-to-expiratory time ratio, airway resistance, and PEEP [14,16]. MP has been suggested as a main determinant of VILI pathogenesis [17-19]. Additionally, it was independently associated with intensive care unit (ICU) mortality, ICU and hospital length of stay, and ventilator-free days in ARDS patients, even when low $\mathrm{V}_{\mathrm{T}}$ and low $\Delta \mathrm{P}$ were applied [20].

In this study, we aimed to assess the changes in MV parameters after the initiation of VV ECMO in a cohort of ARDS patients. The primary aim of this pilot study was to describe and quantify the variation of MP resulting from the adjustment of MV settings. The second aim was to evaluate whether MP was associated with ICU mortality when analyzed during the initial phases of ECMO run.

\section{Materials and Methods}

\subsection{Study Population}

This multi-center, prospective, observational study was performed between December 2015 and June 2017 at three European ICUs experienced with ECMO: Foundation IRCCS San Matteo Hospital (Pavia, Italy), Universitätsklinikum Regensburg (Regensburg, Germany) and Hôpital Erasme (Brussels, Belgium). Institutional review boards of each center approved the study protocol. Eligible candidates were screened from local investigators. Informed consent was acquired retrospectively from every patient or a member of the family, as appropriate [21], according to local laws.

Patients enrolled in this study had severe ARDS according to the Berlin Definition [22], unresponsive to maximal medical therapy. VV ECMO was employed according to Extracorporeal Life Support Organization (ELSO) guidelines for adult respiratory failure [23]. Exclusion criteria were age $<18$ years, mechanical ventilation $\geq 7$ days before ECMO implementation, futility, extracorporeal support as bridge to lung transplantation and involvement in other interventional trials conflicting with the present study. Drop-out criteria were patient extubation during ECMO, relocation to another ICU, unpredicted situations that would not allow detailed evaluation and continuous monitoring (i.e., failing circuits). Patients study recruitment was completed within $12 \mathrm{~h}$ from ECMO initiation. Standard care was provided according to clinical practice.

\subsection{Data Collection}

We registered all data in an anonymous Excel file (Excel 2010, v14.0. Microsoft Corporation, Redmond, WA, USA). After study enrolment, patient demographic and anthropometric data, as well as diagnosis upon hospital and ICU admission, were collected. Sequential Organ Failure Assessment (SOFA) score [24] and Simplified Acute Physiology 
Score (SAPS) II [25] on ICU admission and Respiratory ECMO Survival Prediction RESP (RESP) score [26] before ECMO implementation were also recorded.

MV settings [PEEP $\left(\mathrm{cmH}_{2} \mathrm{O}\right)$, plateau pressure $\left(\mathrm{P}_{\text {plat }}, \mathrm{cmH}_{2} \mathrm{O}\right)$, peak respiratory pressure $\left(\mathrm{P}_{\text {peak }}, \mathrm{cmH}_{2} \mathrm{O}\right), \Delta \mathrm{P}\left(\mathrm{cmH}_{2} \mathrm{O}\right), \mathrm{RR}$ (breaths $\left./ \mathrm{min}\right), \mathrm{V}_{\mathrm{T}}$ adjusted to patient ideal body weight $\left(\mathrm{V}_{\mathrm{T}} / \mathrm{IBW}, \mathrm{mL}\right)$, inspired oxygen fraction $\left(\mathrm{F}_{\mathrm{i}} \mathrm{O}_{2}\right.$, ratio)] were recorded simultaneously, the first time within $12 \mathrm{~h}$ from the initiation of VV ECMO, then once a day during the entire ECMO length. Last reported MV settings before ECMO cannulation were also collected. $\mathrm{P}_{\text {peak }}$ was considered equal to $\mathrm{P}_{\text {plat }}$ in pressure controlled MV modes. $\Delta \mathrm{P}$ was directly computed as the difference between the reported values of $P_{\text {plat }}$ and PEEP [4]. $V_{T} / I B W$ was calculated according to the Devine formula [27]. VV ECMO run and ICU stay lengths were recorded, such as ECMO successful weaning and ICU mortality.

Each MP record was calculated retrospectively from MV data, using an energy calculator, developed by Gattinoni et al. for this specific purpose [28]. We adopted a simplified formula derived from the extended equation [14], as follows:

$$
\text { Power }_{r s}=0.098 \times R R \times V_{T} \times\left(P_{\text {peak }}-\frac{1}{2} \Delta P\right)
$$

MP values were obtained by filling in the software $R R, V_{T}$ and $P_{\text {peak }}$. This mathematical simplification of the original mechanical power formula allows an easier computation of MP at bedside. As stated in the original paper [14], this formula is limited, as the extended one, by the assumption of a linear compliance of the respiratory system in the range of considered pressures and volumes.

\subsection{Statistical Analysis}

Statistical analysis was conducted with STATA [Stata Statistical Software: Release 14 (2015). StataCorp LP, College Station, TX, USA] and significance level was set at 0.05 . Categorical data are expressed as counts and percentage; continuous data are presented as median (IQR, 25th-75th percentiles).

Study patients were evaluated according to the ICU outcome (i.e., non-survivors vs. survivors). Baseline and clinical characteristics of the patients were compared among these groups using Fisher's exact test and Mann Whitney U-test, as appropriate. To estimate the MV variations after ECMO, the mean values of the first $48 \mathrm{~h}$ of ECMO run was considered for each patient. Wilcoxon rank-sum test for paired data was used to test changes in MV parameters before and after ECMO initiation.

The duration of ECMO therapy was divided into quartiles for each patient and the MV variables mean values of the first quartile were considered. As such, MV parameters were analyzed according to the ICU outcome, using the Wilcoxon rank-sum test. Considering the first quartile of ECMO run, MV continuous variables were categorized according to the mean values of our sample. MP value was therefore categorized according to the threshold for risk of VILI from an experimental model (i.e., $12 \mathrm{~J} / \mathrm{min}$ ) [17]. Pearson's chi-square test was then used to analyze the correlation between MV parameters (including MP) and ICU mortality.

\section{Results}

\subsection{Study Population}

From a total of 151 patients undergoing VV ECMO during the study period, 35 patients were included in the final analysis. The most frequent diagnosis was primary ARDS from bacterial $(n=20,57 \%)$ or viral pneumonia $(n=6,17 \%)$. Other common diagnoses were secondary ARDS from abdominal sepsis $(n=3,9 \%)$ and major trauma $(n=2,6 \%)$. Among the remaining patients $(\mathrm{n}=4,11 \%)$, three developed a primary ARDS after fungal pneumonia, lung transplantation and chemotherapy, respectively, while the fourth patient developed ARDS secondary to major surgery.

Median age of the study cohort was 53 (40-64) years; other demographic and anthropometric baseline data among the study population are listed in Table 1. Prognostic scores 
on ICU admission and the mechanical ventilation settings before ECMO initiation are also reported in Table 1. Median length of ICU stay was 20 (11-33) days, while median duration of ECMO run was 10 (4-15) days. ECMO weaning was successful in 28 patients $(80 \%)$; the remaining seven patients died while on ECMO support. Four additional patients died due to complications during the ICU stay after successful ECMO removal; as such, overall ICU mortality was $32 \%$ (11/35 patients).

Table 1 reports the main differences between ICU non-survivors and survivors. SAPS II score [68 (51-80) vs. 49 (37-60), $p=0.005]$ and PEEP before ECMO [10 (8-12) vs. 15 (12-16), $p=0.03$ ] were significantly different between these groups; ICU stay [11 (5-15) vs. 28 (16-38) days, $p=0.009$ ] and duration of ECMO run [4 (2-11) vs. 10 (5-16) days, $p=0.031$ ) were notably shorter in ICU non-survivors when compared to others.

Table 1. Study population baseline demographic and anthropometric characteristics, ICU admission prognostic scores, MV settings before VV ECMO initiation (pre-ECMO), ICU stay and ECMO run length. Variables comparison according to ICU mortality. [ICU intensive care unit, MV mechanical ventilation, VV ECMO veno-venous extracorporeal membrane oxygenation, BMI body mass index, SOFA sequential organ failure assessment, SAPS simplified acute physiology score, RESP respiratory ECMO survival prediction, MP mechanical power, $\mathrm{PEEP}$ positive end-expiratory pressure, $\mathrm{P}_{\text {plat }}$ plateau pressure, $\mathrm{P}_{\text {peak }}$ peak pressure, $\Delta \mathrm{P}$ driving pressure, $\mathrm{RR}$ respiratory rate, $\mathrm{V}_{\mathrm{T}} / \mathrm{IBW}$ patient ideal body weight adjusted tidal volume, $\mathrm{F}_{\mathrm{i}} \mathrm{O}_{2}$ lung inspiratory oxygen fraction].

\begin{tabular}{|c|c|c|c|c|}
\hline & $\begin{array}{l}\text { Study Population } \\
\qquad(\mathrm{n}=35)\end{array}$ & $\begin{array}{l}\text { ICU Non-Survivors } \\
\qquad(\mathrm{n}=11)\end{array}$ & $\begin{array}{l}\text { ICU Survivors } \\
\quad(n=24)\end{array}$ & $p$-Value \\
\hline Age [years; median value (25p-75p)] & $53(40-64)$ & $53(39-67)$ & $53(42-62)$ & 0.902 \\
\hline Male sex [n; \%] & $24(68)$ & $6(54)$ & $18(75)$ & 0.233 \\
\hline Weight $[\mathrm{kg} ;$ median value $(25 \mathrm{p}-75 \mathrm{p})]$ & $84(70-110)$ & $75(60-85)$ & $85(77-118)$ & 0.057 \\
\hline Height $[\mathrm{cm}$; median value (25p-75p)] & $175(169-180)$ & $171(167-177)$ & $177(169-180)$ & 0.182 \\
\hline BMI $\left[\mathrm{kg} / \mathrm{m}^{2} ;\right.$ median value $\left.(25 \mathrm{p}-75 \mathrm{p})\right]$ & $27(24-35)$ & $24(21-29)$ & $28(26-37)$ & 0.145 \\
\hline SOFA score [n; median value (25p-75p)] & $12(9-17)$ & $14(11-18)$ & $12(8-17)$ & 0.228 \\
\hline SAPS II score [n; median value (25p-75p)] & $53(42-68)$ & $68(51-80)$ & $49(37-60)$ & 0.005 \\
\hline RESP score [n; median value (25p-75p)] & $-4(-7-0)$ & $-6(-9--1)$ & $-3(-7-0)$ & 0.398 \\
\hline $\begin{array}{c}\text { pre-ECMO MP } \\
{[\mathrm{J} / \mathrm{min} ; \text { median value }(25 \mathrm{p}-75 \mathrm{p})]}\end{array}$ & $32.4(29.3-36.6)$ & $31.1(29.4-35.8)$ & $32.6(23.6-38.2)$ & 0.918 \\
\hline $\begin{array}{c}\text { pre-ECMO PEEP } \\
{\left[\mathrm{cmH}_{2} \mathrm{O} ; \text { median value }(25 \mathrm{p}-75 \mathrm{p})\right]}\end{array}$ & $14(10-15)$ & $10(8-12)$ & $15(12-16)$ & 0.032 \\
\hline $\begin{array}{c}\text { pre-ECMO } \mathbf{P}_{\text {plat }} \\
{\left[\mathrm{cmH}_{2} \mathrm{O} ; \text { median value }(25 \mathrm{p}-75 \mathrm{p})\right]}\end{array}$ & $27(21-33)$ & $30(19-38)$ & $27(21-31)$ & 0.506 \\
\hline $\begin{array}{c}\text { pre-ECMO } \mathbf{P}_{\text {peak }} \\
{\left[\mathrm{cmH}_{2} \mathrm{O} ; \text { median value }(25 \mathrm{p}-75 \mathrm{p})\right]}\end{array}$ & $33(29-37)$ & $34(30-38)$ & $33(28-35)$ & 0.604 \\
\hline $\begin{array}{c}\text { pre-ECMO } \Delta \mathbf{P} \\
{\left[\mathrm{cmH}_{2} \mathrm{O} ; \text { median value }(25 \mathrm{p}-75 \mathrm{p})\right]}\end{array}$ & $11(7-23)$ & $22(7-29)$ & $13(7-13)$ & 0.237 \\
\hline $\begin{array}{c}\text { pre-ECMO RR } \\
\text { [breaths/min; median value (25p-75p)] }\end{array}$ & $22(20-30)$ & $25(23-40)$ & $21(18-27)$ & 0.059 \\
\hline $\begin{array}{c}\text { pre-ECMO V } \mathbf{V}_{\mathrm{T}} / \mathbf{I B W} \\
{[\mathrm{mL} / \mathrm{kg} ; \text { median value }(25 \mathrm{p}-75 \mathrm{p})]}\end{array}$ & $5.5(4.3-7.4)$ & $5.1(4.1-6.9)$ & $5.9(4.8-7.4)$ & 0.441 \\
\hline $\begin{array}{c}\text { pre-ECMO } \mathrm{F}_{\mathrm{i}} \mathrm{O}_{2} \\
\text { [ratio; median value }(25 \mathrm{p}-75 \mathrm{p})]\end{array}$ & $1.00(0.80-1.00)$ & $1.00(0.80-1.00)$ & $1.00(0.80-1.00)$ & 0.929 \\
\hline $\begin{array}{c}\text { ICU stay length } \\
\text { [days; median value }(25 \mathrm{p}-75 \mathrm{p})]\end{array}$ & $20(11-33)$ & $11(5-15)$ & $28(16-38)$ & 0.009 \\
\hline $\begin{array}{c}\text { VV ECMO run length } \\
\text { [days; median value }(25 p-75 p) \text { ] }\end{array}$ & $10(4-15)$ & $4(2-11)$ & $10(5-16)$ & 0.031 \\
\hline
\end{tabular}




\section{2. $M V$ Parameters before and after ECMO Initiation}

Table 2 and Figure 1 show main differences in MV parameters before and after VV ECMO initiation. In particular, a significant reduction in MP [32.4 (29.3-36.6) vs. 8.2 $(5.5-11.7) \mathrm{J} / \mathrm{min}, p<0.001]$ was observed. Similarly $\mathrm{P}_{\text {plat }}\left[27\right.$ (21-33) vs. $21(20-25) \mathrm{cmH}_{2} \mathrm{O}$, $p=0.012], \mathrm{P}_{\text {peak }}\left[33(29-37)\right.$ vs. $\left.30(21-32) \mathrm{cmH}_{2} \mathrm{O}, p<0.001\right], \Delta \mathrm{P}[11(7-23)$ vs. $8(7-10)$ $\mathrm{cmH}_{2} \mathrm{O}, p=0.014$ ], RR [22 (20-30) vs. 14 (10-17) breaths/min, $p<0.001$ ], $\mathrm{V}_{\mathrm{T}} / \mathrm{IBW}$ [5.5 $(4.3-7.4)$ vs. $4.0(2.8-5.4 \mathrm{~mL} / \mathrm{kg}, p=0.001]$ and $\mathrm{F}_{\mathrm{i}} \mathrm{O}_{2}[1.0(0.80-1.00)$ vs. $0.60(0.40-0.80)$, $p<0.001]$ significantly decreased after ECMO initiation.

Table 2. Differences in MV parameters before (pre-ECMO) and during the first $48 \mathrm{~h}$ after VV ECMO initiation (ECMO). [MV mechanical ventilation, VV ECMO veno-venous extracorporeal membrane oxygenation, $\triangle \%$ percentage variation, MP mechanical power, $\mathrm{PEEP}$ positive end-expiratory pressure, $\mathrm{P}_{\text {plat }}$ plateau pressure, $\mathrm{P}_{\text {peak }}$ peak pressure, $\Delta \mathrm{P}$ driving pressure, $\mathrm{RR}$ respiratory rate, $\mathrm{V}_{\mathrm{T}}$ /IBW patient ideal body weight adjusted tidal volume, $\mathrm{F}_{\mathrm{i}} \mathrm{O}_{2}$ lung inspiratory oxygen fraction].

\begin{tabular}{|c|c|c|c|c|}
\hline & $\begin{array}{c}\text { pre-ECMO } \\
(\mathrm{n}=35)\end{array}$ & $\begin{array}{l}\text { ECMO } \\
(n=35)\end{array}$ & $\Delta \%$ & $p$-Value \\
\hline MP [J/min; median value (25p-75p)] & $32.4(29.3-36.6)$ & $8.2(5.5-11.7)$ & $-74.7 \%$ & $<0.001$ \\
\hline PEEP $\left[\mathrm{cmH}_{2} \mathrm{O} ;\right.$ median value $\left.(25 \mathrm{p}-75 \mathrm{p})\right]$ & $14(10-15)$ & $13(10-16)$ & $-7.1 \%$ & 0.390 \\
\hline $\mathbf{P}_{\text {plat }}\left[\mathrm{cmH}_{2} \mathrm{O} ;\right.$ median value $\left.(25 \mathrm{p}-75 \mathrm{p})\right]$ & $27(21-33)$ & $21(20-25)$ & $-22.2 \%$ & 0.012 \\
\hline $\mathbf{P}_{\text {peak }}\left[\mathrm{cmH}_{2} \mathrm{O} ;\right.$ median value $\left.(25 p-75 p)\right]$ & $33(29-37)$ & $30(21-32)$ & $-9.1 \%$ & $<0.001$ \\
\hline$\Delta \mathbf{P}\left[\mathrm{cmH}_{2} \mathrm{O} ;\right.$ median value $\left.(25 \mathrm{p}-75 \mathrm{p})\right]$ & $11(7-23)$ & $8(7-10)$ & $-27.3 \%$ & 0.014 \\
\hline RR [breaths/min; median value (25p-75p)] & $22(20-30)$ & $14(10-17)$ & $-36.4 \%$ & $<0.001$ \\
\hline $\mathbf{V}_{\mathrm{T}} / \mathrm{IBW}[\mathrm{mL} / \mathrm{kg} ;$ median value (25p-75p)] & $5.5(4.3-7.4)$ & $4.0(2.8-5.4)$ & $-27.3 \%$ & 0.001 \\
\hline $\mathbf{F}_{\mathbf{i}} \mathbf{O}_{2}$ [ratio; median value (25p-75p)] & $1.00(0.80-1.00)$ & $\mathbf{0 . 6 0}(0.40-0.80)$ & $-40.0 \%$ & $<0.001$ \\
\hline
\end{tabular}

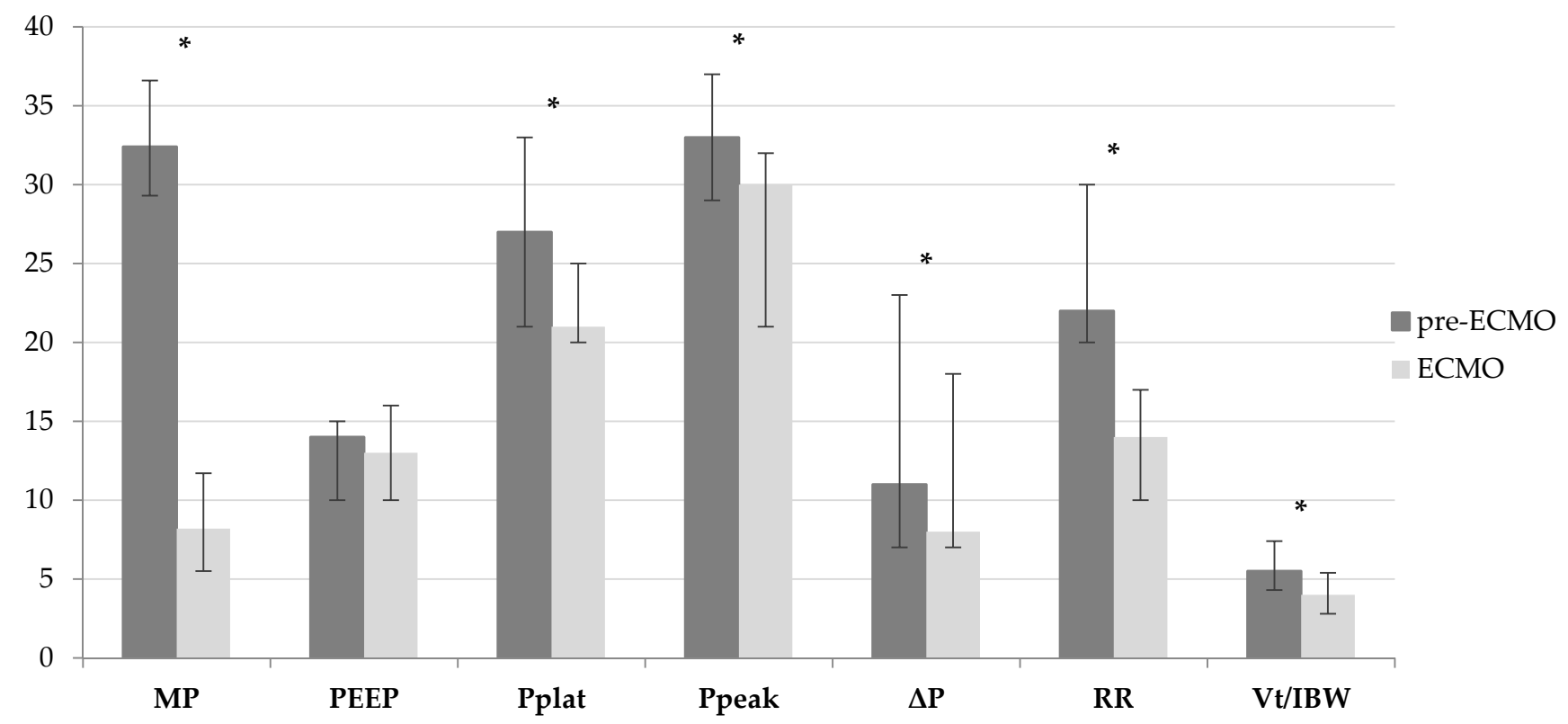

Figure 1. Differences in MV parameters before (pre-ECMO) and during the first $48 \mathrm{~h}$ after VV ECMO initiation (ECMO). [MV mechanical ventilation, VV ECMO veno-venous extracorporeal membrane oxygenation, MP mechanical power, PEEP positive end-expiratory pressure, $\mathrm{P}_{\text {plat }}$ plateau pressure, $\mathrm{P}_{\text {peak }}$ peak pressure, $\Delta \mathrm{P}$ driving pressure, $\mathrm{RR}$ respiratory rate, $\mathrm{V}_{\mathrm{T}}$ /IBW patient ideal body weight adjusted tidal volume]. 


\subsection{Parameters during Early Phases of ECMO Run and ICU Mortality}

As shown in Table 3, during the first quartile of ECMO run, RR [17 (15-25) vs. 13 (10-16) breaths $/ \mathrm{min}, p=0.003$ ] was significantly higher in ICU non-survivors than survivors. Similarly, PEEP [10 (8-12) vs. $\left.14(11-16) \mathrm{cmH}_{2} \mathrm{O}, p=0.048\right]$ and $\mathrm{V}_{\mathrm{T}} / \mathrm{IBW}[3.0$ $(2.0-4.0)$ vs. $4.0(3.5-6.0) \mathrm{mL} / \mathrm{kg}, p=0.028]$ were significantly lower in ICU non-survivors. Moreover, a RR greater than 15 breaths/min correlated to an increase in ICU mortality ( $p=0.008$, Table 4). No further differences in other MV variables values (including MP) were detected between these groups.

Table 3. Comparison of MV parameters during the first quartile of VV ECMO length, according to the ICU mortality. [MV mechanical ventilation, VV ECMO veno-venous extracorporeal membrane oxygenation, ICU intensive care unit, MP mechanical power, $\mathrm{PEEP}$ positive end-expiratory pressure, $\mathrm{P}_{\text {plat }}$ plateau pressure, $\mathrm{P}_{\text {peak }}$ peak pressure, $\Delta \mathrm{P}$ driving pressure, $\mathrm{RR}$ respiratory rate, $\mathrm{V}_{\mathrm{T}}$ /IBW patient ideal body weight adjusted tidal volume, $\mathrm{F}_{\mathrm{i}} \mathrm{O}_{2}$ lung inspiratory oxygen fraction].

\begin{tabular}{|c|c|c|c|c|}
\hline & $\begin{array}{l}\text { Study Population } \\
\qquad(\mathrm{n}=35)\end{array}$ & $\begin{array}{l}\text { ICU Non-Survivors } \\
\quad(n=11)\end{array}$ & $\begin{array}{l}\text { ICU Survivors } \\
\quad(n=24)\end{array}$ & $p$-Value \\
\hline MP [J/min; median value (25p-75p)] & $8.0(5.0-14.0)$ & $8.0(5.0-20.0)$ & $8.0(6.0-13.0)$ & 0.530 \\
\hline PEEP $\left[\mathrm{cmH}_{2} \mathrm{O}\right.$; median value (25p-75p)] & $12(10-16)$ & $10(8-12)$ & $14(11-16)$ & 0.048 \\
\hline $\mathbf{P}_{\text {plat }}\left[\mathrm{cmH}_{2} \mathrm{O} ;\right.$ median value $\left.(25 \mathrm{p}-75 \mathrm{p})\right]$ & $22(20-25)$ & $22(19-29)$ & $22(20-25)$ & 0.880 \\
\hline $\mathbf{P}_{\text {peak }}\left[\mathrm{cmH}_{2} \mathrm{O} ;\right.$ median value $\left.(25 \mathrm{p}-75 \mathrm{p})\right]$ & $26(22-31)$ & $30(24-34)$ & $26(21-30)$ & 0.174 \\
\hline$\Delta \mathbf{P}\left[\mathrm{cmH}_{2} \mathrm{O} ;\right.$ median value $\left.(25 \mathrm{p}-75 \mathrm{p})\right]$ & $10(7-12)$ & $12(7-15)$ & $9(6-12)$ & 0.229 \\
\hline $\begin{array}{l}\text { RR [breaths/min; median value } \\
\qquad(25 p-75 p)]\end{array}$ & $14(10-17)$ & $17(15-25)$ & $13(10-16)$ & 0.003 \\
\hline $\begin{array}{c}\mathbf{V}_{\mathbf{T}} / \text { IBW }[\mathrm{mL} / \mathrm{kg} ; \text { median value } \\
(25 \mathrm{p}-75 \mathrm{p})]\end{array}$ & $4.0(3.0-5.0)$ & $3.0(2.0-4.0)$ & $4.0(3.5-6.0)$ & 0.028 \\
\hline $\mathrm{F}_{\mathrm{i}} \mathrm{O}_{2}$ [ratio; median value (25p-75p)] & $0.55(0.43-0.73)$ & $0.43(0.40-0.73)$ & $0.57(0.50-0.75)$ & 0.345 \\
\hline
\end{tabular}

Table 4. Association with ICU mortality, according to the categorization of MV parameters during the first quartile of VV ECMO run. [ICU intensive care unit, MV mechanical ventilation, VV ECMO venovenous extracorporeal membrane oxygenation, MP mechanical power, PEEP positive end-expiratory pressure, $\mathrm{P}_{\text {plat }}$ plateau pressure, $\mathrm{P}_{\text {peak }}$ peak pressure, $\Delta \mathrm{P}$ driving pressure, $\mathrm{RR}$ respiratory rate, $\mathrm{V}_{\mathrm{T}}$ /IBW patient ideal body weight adjusted tidal volume, $\mathrm{F}_{\mathrm{i}} \mathrm{O}_{2}$ lung inspiratory oxygen fraction].

\begin{tabular}{cccc}
\hline & $\begin{array}{c}\text { Variable } \\
\text { Categorization }\end{array}$ & $\begin{array}{c}\text { Pearson's } \\
\text { Chi-Square Test }\end{array}$ & $p$-Value \\
\hline $\mathbf{M P}(\mathrm{J} / \mathrm{min})$ & $\leq 12 ;>12$ & 0.475 & 0.491 \\
\hline $\mathbf{P E E P}\left(\mathrm{cmH}_{2} \mathrm{O}\right)$ & $<12 ; \geq 12$ & 2.828 & 0.093 \\
\hline $\mathbf{P}_{\text {plat }}\left(\mathrm{cmH}_{2} \mathrm{O}\right)$ & $\leq 23 ;>23$ & 2.198 & 0.138 \\
\hline $\mathbf{P}_{\text {peak }}\left(\mathrm{cmH}_{2} \mathrm{O}\right)$ & $\leq 27 ;>27$ & 0.957 & 0.328 \\
\hline $\boldsymbol{\Delta P}\left(\mathrm{cmH}_{2} \mathrm{O}\right)$ & $<10 ; \geq 10$ & 1.247 & 0.264 \\
\hline $\mathbf{R} \mathbf{R}(\mathrm{breaths} / \mathrm{min})$ & $<15 ; \geq 15$ & 7.098 & 0.008 \\
\hline $\mathbf{V}_{\mathbf{T}} / \mathbf{I B W}(\mathrm{mL} / \mathrm{kg})$ & $<4.5 ; \geq 4.5$ & 1.847 & 0.174 \\
\hline $\mathbf{F}_{\mathbf{i}} \mathbf{O}_{\mathbf{2}}[\mathrm{ratio}]$ & $\leq 0.6 ;>0.6$ & 0.088 & 0.766 \\
\hline
\end{tabular}

\section{Discussion}

In this pilot study, we evaluated MP and other MV parameters after VV ECMO initiation and its prognostic role in a selected ARDS population. We observed that an ultra-protective lung ventilation strategy was applied in these patients, with a consequent significant reduction in MP after ECMO initiation. Nevertheless, RR, PEEP, and $\mathrm{V}_{\mathrm{T}} / \mathrm{IBW}$, 
but not MP, were the MV variables that differed during the early phases of ECMO run between ICU non-survivors and survivors.

During ARDS, MV is grounded on minimizing $\mathrm{V}_{\mathrm{T}}$, with a reduction in $\triangle \mathrm{P}$, and maintaining adequate level of PEEP [6]. Nowadays, it is possible to safely rely on VV ECMO support for both oxygenation and carbon dioxide clearance, limiting VILI and allowing the lungs to recover $[7,12]$. Despite lung-protective ventilation being relatively standardized in ARDS patients without ECMO [29], there is no specific recommendation on how to manage native lung ventilation and respiratory workload during VV ECMO [30,31], which resulted into different strategies in clinical practice $[3,32,33]$. As for all ARDS patients, $\triangle \mathrm{P}$ has been shown to be an important MV variable correlating with mortality in VV ECMO [34-36]; however, there are no large studies showing the effects of $\Delta \mathrm{P}$-individualized MV therapy in ECMO patients and lung recovery or patients' survival [37].

In the last years, the severity of VILI has been related to the MP, which represents the amount of energy transmitted during MV to the respiratory system per time unit [14]. Taking into account some potential limitations [15,38,39], MP might represent a useful tool to optimize MV and potentially limit VILI [16-19] during ECMO. In our multi-center observational study, the median pre-ECMO MP value of $32.4 \mathrm{~J} / \mathrm{min}$ was particularly high, considering the threshold (i.e., $17.0 \mathrm{~J} / \mathrm{min}$ ), which has been associated with an increased risk of mortality [20]. This is an interesting finding, since it reported the inadequacy of MV settings in severe ARDS patients failing to respond to conventional therapies [3,4]. After MV adjustments following ECMO initiation, the median MP value dropped significantly to $8.2 \mathrm{~J} / \mathrm{min}$, which is below the reported threshold (i.e., $12 \mathrm{~J} / \mathrm{min}$ ) which was associated with an increased risk of VILI in experimental models [17]. This reduction in MP is consistent with results from an international study from experienced ECMO centers [37]. Taking into account MV settings, our results showed that ultra-protective ventilation strategy [32], with significant reductions in $\mathrm{V}_{\mathrm{T}} / \mathrm{IBW}$ (from 5.5 to $4.0 \mathrm{~mL} / \mathrm{kg}$ ), $\mathrm{P}_{\text {plat }}$ (from 27 to $21 \mathrm{cmH}_{2} \mathrm{O}$ ), $\Delta \mathrm{P}$ (from 11 to $8 \mathrm{cmH}_{2} \mathrm{O}$ ) and $\mathrm{RR}$ (from 22 to 14 breaths/min) was feasible in all patients after ECMO initiation. However, lung ventilation parameters were also within the "protective" ranges before ECMO initiation [3], with levels of PEEP (from 14 to $13 \mathrm{cmH}_{2} \mathrm{O}$ ) indicating the maintenance of an "open lung" strategy [40,41], even during ECMO. These findings suggest the importance of MP monitoring at the bedside, as lung stress may occur even within acceptable ranges of $\mathrm{V}_{\mathrm{T}}, \mathrm{P}_{\text {plat }}$, and PEEP and might prompt an earlier use of extra-corporeal therapies to reduce the occurrence of VILI. Whether high PEEP levels, which are relevant to avoid alveolar de-recruitment during ECMO [10,42], can also influence patients' outcome remains to be demonstrated; also, individualized PEEP levels using MP monitoring or other techniques $[43,44]$ remains a challenging issue that requires further investigation.

In this study, the ICU mortality (32\%) was comparable to other reports [37]. According to the baseline data of our population, the higher SAPS II score in non-survivors (68 vs. 49) suggested a possible negative effect on outcomes of extra-pulmonary organ failure before VV ECMO. However, median SAPS II values were below the threshold of 80, which has been suggested as an indicator of poor outcome in ECMO patients [45,46]. This finding confirms the need for a complete evaluation of patients' conditions before ECMO and underlines the utility of prognostic scores to identify patients who are more likely to benefit from ECMO therapy.

The comparison of MV parameters between ICU non-survivors and survivors was limited to the first quartile of ECMO length for each patient, because this period requires the maximal effort to optimize lung protection and to limit VILI [6,47]. Later on during the ECMO run, when healing of lung parenchyma takes place, a less-protective approach is possible and efforts can be directed to promote ECMO weaning $[48,49]$. The lack of association between early MP changes and mortality is not inconsistent with this approach. Mortality during ECMO is not only due to persistent lung injury but also determined by secondary complications (i.e., bleeding, sepsis, acute ischemic stroke) and decisions to withdraw life-sustaining therapies. Nonetheless, MP should not be overlooked while on ECMO. Other MV parameters that account for MP computation, such as PEEP, $\mathrm{V}_{\mathrm{T}} / \mathrm{IBW}$, 
and RR should also be carefully monitored. The lower PEEP values in ICU non-survivors (10 vs. $14 \mathrm{cmH}_{2} \mathrm{O}$ ) could support the open lung strategy [40-43]. At the same time, the lower $\mathrm{V}_{\mathrm{T}} / \mathrm{IBW}$ in ICU deaths $(3.0$ vs. $4.0 \mathrm{~mL} / \mathrm{kg})$ might suggest a limit value in $\mathrm{V}_{\mathrm{T}}$ reduction, even on ECMO $[9,50,51]$. We also observed differences in ICU mortality when RR was categorized according to a specific threshold (i.e., 15 breaths $/ \mathrm{min}$ ). In clinical studies, RR has progressively received more attention, gaining relevance over $V_{T}$ and airways pressures on VILI prevention and limitation [52]. A near-apneic MV (RR 5 breaths $/ \mathrm{min}$ ) revealed decreased lung injury in ARDS patients treated with VV ECMO [53]. Furthermore, while maintaining other $\mathrm{MV}$ parameters stable $\left(\mathrm{P}_{\text {plat }}, \Delta \mathrm{P}, \mathrm{V}_{\mathrm{T}}, \mathrm{PEEP}\right)$, an animal model showed for each 5-fold increase in RR an 11-fold increase in MP [17]. To date, the literature is still lacking in well-defined clinical recommendations concerning RR for MV adjustment during VV ECMO [23,54,55]. Our results corroborate the crucial role of the duration of exposure to the delivered injuring strain (i.e., numbers of cycles) to determine thresholds [56], highlighting RR as one of the main determinants for energy transmission $[18,30]$.

\section{Study Limitations}

This pilot study has several limitations, mostly due to the small sample size. As a consequence, we had a limited number of observations to run a multivariable model to assess independent predictors of ICU mortality. We involved three different experienced ECMO centers, with specific patient selection criteria, which could reduce generalizability of overall findings. For practical issues, MV parameters and settings were only collected once a day and may thus not properly describe all the potential settings changes in a $24-h$ period, especially in the first stages of ECMO run. For this reason, we evaluated the MV variations after ECMO initiation taking into account, for each patient, the mean values of the first $48 \mathrm{~h}$ of ECMO length. Furthermore, our population showed a significant variability among ECMO run duration, with a mean value of 10 days and a standard deviation of 8 days. We believe that the correlation analysis between MV settings and ICU mortality based on the first quartiles of ECMO length for each patient, rather than on a fixed number of days (as presented in other VV ECMO studies), better mirrors the actual acute phase of ARDS for a given patient. Lastly, we would underline that all the MP values were calculated from the MV parameters' datasheet.

\section{Conclusions}

There are no recommendations on optimal MV settings during VV ECMO. The results from this pilot study confirmed that VV ECMO allows a significant reduction of MP. Early MP values did not predict patients' outcome in this cohort. Further larger studies are needed to assess the prognostic role of MP and other MV parameters in VV ECMO patients. Importantly, these parameters should still be adequately monitored in this setting.

Author Contributions: M.B., M.V.M. and F.S.T. promoted and supervised the study. M.B., F.E. and L.C. were involved in the conception and design of the study and wrote the manuscript. L.C., F.E. and C.F. collected data and completed the study database. M.G.Q. and F.F. performed data analysis and statically revision. M.B., M.V.M., F.S.T., F.F., M.D.N., L.M.B. and R.L. contributed with important intellectual content and revision of the manuscript. All authors have read and agreed to the published version of the manuscript.

Funding: The publication fee was supported by Fondazione IRCCS Policlinico San Matteo, Pavia, Italy. Institutional Review Board Statement: Not applicable.

Informed Consent Statement: Not applicable.

Acknowledgments: The authors would like to thank the staff members of the ICUs involved in this study, who contributed to data sampling and reporting. The authors are grateful to Associate Professor Peter Radell (Pediatric Intensive Care, Astrid Lindgren Children's Hospital, Karolinska University Hospital, Stockholm, Sweden) for English language editing. 
Conflicts of Interest: M.B. is a congress speaker for Hamilton Medical and advisor for Eurosets. M.V.M. is a consultant or speaker for Medtronic, Getinge and Xenios, and advisor for Eurosets. F.S.T. and M.D.N. are advisors for Eurosets. L.M.B. is an advisor for Eurosets and Xenios. R.L. is a consultant for Medtronic and LivaNova, and advisor for Eurosets (all honoraria paid to the university). F.E., L.C., F.F., M.G.Q. and C.F. declare no conflict of interest. The funders had no role in the design of the study; in the collection, analyses, or interpretation of data; in the writing of the manuscript, or in the decision to publish the results.

\section{References}

1. Rezoagli, E.; Fumagalli, R.; Bellani, G. Definition and epidemiology of acute respiratory distress syndrome. Ann. Transl. Med. 2017, 5, 282. [CrossRef] [PubMed]

2. Gattinoni, L.; Pesenti, A. The concept of "baby lung". Intensive Care Med. 2005, 31, 776-784. [CrossRef] [PubMed]

3. Network, A.R.D.S.; Brower, R.G.; Matthay, M.A.; Morris, A.; Schoenfeld, D.; Thompson, B.T.; Wheeler, A. Ventilation with Lower Tidal Volumes as Compared with Traditional Tidal Volumes for Acute Lung Injury and the Acute Respiratory Distress Syndrome. N. Engl. J. Med. 2000, 342, 1301-1308. [CrossRef]

4. Amato, M.B.P.; Meade, M.O.; Slutsky, A.S.; Brochard, L.; Costa, E.L.V.; Schoenfeld, D.A.; Stewart, T.E.; Briel, M.; Talmor, D.S.; Mercat, A.; et al. Driving Pressure and Survival in the Acute Respiratory Distress Syndrome. N. Engl. J. Med. 2015, 372, 747-755. [CrossRef]

5. Bugedo, G.; Retamal, J.; Bruhn, A. Driving pressure: A marker of severity, a safety limit, or a goal for mechanical ventilation? Crit. Care 2017, 21, 1-7. [CrossRef]

6. Papazian, L.; Aubron, C.; Brochard, L.; Chiche, J.-D.; Combes, A.; Dreyfuss, D.; Forel, J.M.; Guérin, C.; Jaber, S.; Dessap, A.M.; et al. Formal guidelines: Management of acute respiratory distress syndrome. Ann. Intensive Care 2019, 9, 69. [CrossRef]

7. Parekh, M.; Abrams, D.; Brodie, D. Extracorporeal techniques in acute respiratory distress syndrome. Ann. Transl. Med. 2017, 5, 296. [CrossRef]

8. Gattinoni, L.; Vasques, F.; Quintel, M. Use of ECMO in ARDS: Does the EOLIA trial really help? Crit. Care 2018, 22, 171. [CrossRef]

9. Rozencwajg, S.; Guihot, A.; Franchineau, G.; Lescroat, M.; Bréchot, N.; Hékimian, G.; Lebreton, G.; Autran, B.; Luyt, C.-E.; Combes, A.; et al. Ultra-Protective Ventilation Reduces Biotrauma in Patients on Venovenous Extracorporeal Membrane Oxygenation for Severe Acute Respiratory Distress Syndrome. Crit. Care Med. 2019, 47, 1505-1512. [CrossRef]

10. Gattinoni, L.; Tonetti, T.; Moerer, O. How best to set the ventilator on extracorporeal membrane lung oxygenation. Curr. Opin. Crit. Care 2017, 23, 66-72. [CrossRef]

11. Gattinoni, L.; Kolobow, T.; Tomlinson, T.; Iapichino, G.; Samaja, M.; White, D.; Pierce, J. Low-Frequency Positive Pressure Ventilation with Extracorporeal Carbon Dioxide Removal (LFPPV-ECCO2R). Anesth. Analg. 1978, 57, 470-477. [CrossRef] [PubMed]

12. Del Sorbo, L.; Goffi, A.; Tomlinson, G.; Pettenuzzo, T.; Facchin, F.; Vendramin, A.; Goligher, E.C.; Cypel, M.; Slutsky, A.S.; Keshavjee, S.; et al. Effect of Driving Pressure Change During Extracorporeal Membrane Oxygenation in Adults with Acute Respiratory Distress Syndrome: A Randomized Crossover Physiologic Study. Crit. Care Med. 2020, 48, 1771-1778. [CrossRef] [PubMed]

13. Hubmayr, R.D.; Kallet, R.H. Understanding Pulmonary Stress-Strain Relationships in Severe ARDS and Its Implications for Designing a Safer Approach to Setting the Ventilator. Respir. Care 2018, 63, 219-226. [CrossRef] [PubMed]

14. Gattinoni, L.; Tonetti, T.; Cressoni, M.; Cadringher, P.; Herrmann, P.; Moerer, O.; Protti, A.; Gotti, M.; Chiurazzi, C.; Carlesso, E.; et al. Ventilator-related causes of lung injury: The mechanical power. Intensive Care Med. 2016, 42, 1567-1575. [CrossRef] [PubMed]

15. Silva, P.L.; Ball, L.; Rocco, P.R.; Pelosi, P. Power to mechanical power to minimize ventilator-induced lung injury? Intensive Care Med. Exp. 2019, 7, 1-11. [CrossRef]

16. Giosa, L.; Busana, M.; Pasticci, I.; Bonifazi, M.; Macrì, M.M.; Romitti, F.; Vassalli, F.; Chiumello, D.; Quintel, M.; Marini, J.J.; et al. Mechanical power at a glance: A simple surrogate for volume-controlled ventilation. Intensive Care Med. Exp. 2019, 7, 1-13. [CrossRef]

17. Cressoni, M.; Gotti, M.; Chiurazzi, C.; Massari, D.; Algieri, I.; Amini, M.; Cammaroto, A.; Brioni, M.; Montaruli, C.; Nikolla, K.; et al. Mechanical Power and Development of Ventilator-induced Lung Injury. Anesthesiology 2016, 124, 1100-1108. [CrossRef]

18. Tonetti, T.; Vasques, F.; Rapetti, F.; Maiolo, G.; Collino, F.; Romitti, F.; Camporota, L.; Cressoni, M.; Cadringher, P.; Quintel, M.; et al. Driving pressure and mechanical power: New targets for VILI prevention. Ann. Transl. Med. 2017, 5, 286. [CrossRef]

19. Marini, J.J.; Rocco, P.R.M.; Gattinoni, L. Static and Dynamic Contributors to Ventilator-induced Lung Injury in Clinical Practice. Pressure, Energy, and Power. Am. J. Respir. Crit. Care Med. 2020, 201, 767-774. [CrossRef]

20. Neto, A.S.; for the PROVE Network Investigators; Deliberato, R.O.; Johnson, A.E.W.; Bos, L.D.; Amorim, P.; Pereira, S.M.; Cazati, D.C.; Cordioli, R.L.; Correa, T.D.; et al. Mechanical power of ventilation is associated with mortality in critically ill patients: An analysis of patients in two observational cohorts. Intensive Care Med. 2018, 44, 1914-1922. [CrossRef]

21. World Medical Association. WMA Declaration of Lisbon on the Rights of the Patient. WMA Declar. 2015. Available online: https:/ / www.wma.net/policies-post/wma-declaration-of-lisbon-on-the-rights-of-the-patient (accessed on 14 November 2020). 
22. Force, A.D.T.; Ranieri, V.M.; Rubenfeld, G.; Thompson, B.T.; Ferguson, N.D.; Caldwell, E.; Fan, E.; Camporota, L.; Slutsky, A.S. Acute Respiratory Distress Syndrome. JAMA 2012, 307, 2526-2533. [CrossRef]

23. Extracorporeal Life Support Organization ELSO Guidelines for Adult Respiratory Failure, Version 1.4. August 2017, pp. 1-32. Available online: https://www.elso.org/Portals/0/ELSO\%20Guidelines\%20For\%20Adult\%20Respiratory\%20Failure\%201_4.pdf (accessed on 14 November 2020).

24. Vincent, J.-L.; Moreno, R.; Takala, J.; Willatts, S.; De Mendonça, A.; Bruining, H.; Reinhart, C.K.; Suter, P.M.; Thijs, L.G. The SOFA (Sepsis-related Organ Failure Assessment) score to describe organ dysfunction/failure. Intensive Care Med. 1996, 22, 707-710. [CrossRef] [PubMed]

25. Le Gall, J.R. A new Simplified Acute Physiology Score (SAPS II) based on a European/North American multicenter study. JAMA 1993, 270, 2957-2963. [CrossRef] [PubMed]

26. Schmidt, M.; Bailey, M.; Sheldrake, J.; Hodgson, C.; Aubron, C.; Rycus, P.T.; Scheinkestel, C.; Cooper, D.J.; Brodie, D.; Pellegrino, V.; et al. Predicting Survival after Extracorporeal Membrane Oxygenation for Severe Acute Respiratory Failure. The Respiratory Extracorporeal Membrane Oxygenation Survival Prediction (RESP) Score. Am. J. Respir. Crit. Care Med. 2014, 189, 1374-1382. [CrossRef] [PubMed]

27. Barnes, T.; Zochios, V.; Parhar, K.K.S. Re-examining Permissive Hypercapnia in ARDS. Chest 2018, 154, 185-195. [CrossRef] [PubMed]

28. Gattinoni, L.; Quintel, M.; Tonetti, T.; Herrmann, P. Energy Calculator Vers. 1.2.6 by P. Herrman. Available online: http: //www.ains.med.uni-goettingen.de/de/abteilung-anaesthesiologie/forschung/energy-calculator-software (accessed on 17 January 2019).

29. Marhong, J.D.; Telesnicki, T.; Munshi, L.; Del Sorbo, L.; Detsky, M.; Fan, E. Mechanical Ventilation during Extracorporeal Membrane Oxygenation. An International Survey. Ann. Am. Thorac. Soc. 2014, 11, 956-961. [CrossRef] [PubMed]

30. Moerer, O.; Busana, M.; Gattinoni, L. Breathing and Ventilation during Extracorporeal Membrane Oxygenation: How to Find the Balance between Rest and Load. Am. J. Respir. Crit. Care Med. 2019, 200, 954-956. [CrossRef]

31. Jenks, C.L.; Tweed, J.; Gigli, K.H.; Venkataraman, R.; Raman, L. An International Survey on Ventilator Practices Among Extracorporeal Membrane Oxygenation Centers. ASAIO J. 2017, 63, 787-792. [CrossRef]

32. Terragni, P.P.; Del Sorbo, L.; Mascia, L.; Urbino, R.; Martin, E.L.; Birocco, A.; Faggiano, C.; Quintel, M.; Gattinoni, L.; Ranieri, V.M. Tidal Volume Lower than $6 \mathrm{ml} / \mathrm{kg}$ Enhances Lung Protection. Anesthesiol. 2009, 111, 826-835. [CrossRef]

33. Swol, J.; Fülling, Y.; Ull, C.; Bechtel, J.M.; Schildhauer, T.A. 48 h cessation of mechanical ventilation during venovenous extracorporeal membrane oxygenation in severe trauma: A case report. J. Artif. Organs 2017, 20, 280-284. [CrossRef]

34. Neto, A.S.; The ReVA Research Network and the PROVE Network Investigators; Schmidt, M.; Azevedo, L.C.P.; Bein, T.; Brochard, L.; Beutel, G.; Combes, A.; Costa, E.L.V.; Hodgson, C.; et al. Associations between ventilator settings during extracorporeal membrane oxygenation for refractory hypoxemia and outcome in patients with acute respiratory distress syndrome: A pooled individual patient data analysis. Intensive Care Med. 2016, 42, 1672-1684. [CrossRef] [PubMed]

35. Gupta, E.; Awsare, B.; Hiroshi, H.; Cavarocchi, N.; Baram, M. Don't Drive Blind: Driving Pressure to Optimize Ventilator Management in ECMO. Lung 2020, 198, 1-8. [CrossRef]

36. Magunia, H.; Haeberle, H.A.; Henn, P.; Mehrländer, M.; Vlatten, P.O.; Mirakaj, V.; Rosenberger, P.; Koeppen, M. Early Driving Pressure Changes Predict Outcomes during Venovenous Extracorporeal Membrane Oxygenation for Acute Respiratory Distress Syndrome. Crit. Care Res. Pract. 2020, 2020, 6958152. [CrossRef] [PubMed]

37. Schmidt, M.; Pham, T.; Arcadipane, A.; Agerstrand, C.; Ohshimo, S.; Pellegrino, V.; Vuylsteke, A.; Guervilly, C.; McGuinness, S.; Pierard, S.; et al. Mechanical Ventilation Management during Extracorporeal Membrane Oxygenation for Acute Respiratory Distress Syndrome. An International Multicenter Prospective Cohort. Am. J. Respir. Crit. Care Med. 2019, 200, $1002-1012$. [CrossRef]

38. Collino, F.; Rapetti, F.; Vasques, F.; Maiolo, G.; Tonetti, T.; Romitti, F.; Niewenhuys, J.; Behnemann, T.; Camporota, L.; Hahn, G.; et al. Positive End-expiratory Pressure and Mechanical Power. Anesthesiol. 2019, 130, 119-130. [CrossRef]

39. Huhle, R.; Neto, A.S.; Schultz, M.J.; De Abreu, M.G. Is mechanical power the final word on ventilator-induced lung injury?-No. Ann. Transl. Med. 2018, 6, 394. [CrossRef]

40. Lachmann, B. (Burkhard) Open up the lung and keep the lung open. Intensive Care Med. 1992, 18, 319-321. [CrossRef]

41. Van Der Zee, P.; Gommers, D. Recruitment Maneuvers and Higher PEEP, the So-Called Open Lung Concept, in Patients with ARDS. Crit. Care 2019, 23, 73. [CrossRef]

42. Protti, A.; Andreis, D.T.; Monti, M.; Santini, A.; Sparacino, C.C.; Langer, T.; Votta, E.; Gatti, S.; Lombardi, L.; Leopardi, O.; et al. Lung Stress and Strain During Mechanical Ventilation. Crit. Care Med. 2013, 41, 1046-1055. [CrossRef]

43. Van Der Zee, P.; Miranda, D.R.; Meeder, H.; Endeman, H.; Gommers, D. vvECMO can be avoided by a transpulmonary pressure guided open lung concept in patients with severe ARDS. Crit. Care 2019, 23, 133. [CrossRef]

44. Camporota, L.; Caricola, E.V.; Bartolomeo, N.; Di Mussi, R.; Wyncoll, D.L.A.; Meadows, C.I.S.; Amado-Rodriguez, L.; Vasques, F.; Sanderson, B.; Glover, G.W.; et al. Lung Recruitability in Severe Acute Respiratory Distress Syndrome Requiring Extracorporeal Membrane Oxygenation. Crit. Care Med. 2019, 47, 1177-1183. [CrossRef] [PubMed] 
45. Kim, K.I.; Lee, H.S.; Kim, H.S.; Ha, S.O.; Lee, W.Y.; Park, S.J.; Lee, S.H.; Lee, T.H.; Seo, J.Y.; Choi, H.H.; et al. The pre-ECMO simplified acute physiology score II as a predictor for mortality in patients with initiation ECMO support at the emergency department for acute circulatory and/or respiratory failure: A retrospective study. Scand. J. Trauma Resusc. Emerg. Med. 2015, 23, 59. [CrossRef] [PubMed]

46. Choi, M.J.; Ha, S.O.; Kim, H.S.; Park, S.; Han, S.J.; Lee, S.H. The Simplified Acute Physiology Score II as a Predictor of Mortality in Patients Who Underwent Extracorporeal Membrane Oxygenation for Septic Shock. Ann. Thorac. Surg. 2017, 103, $1246-1253$. [CrossRef] [PubMed]

47. Matthay, M.A.; Zemans, R.L.; Zimmerman, G.A.; Arabi, Y.M.; Beitler, J.R.; Mercat, A.; Herridge, M.; Randolph, A.G.; Calfee, C.S. Acute respiratory distress syndrome. Nat. Rev. Dis. Prim. 2019, 5, 1-22. [CrossRef]

48. Grant, A.A.; Hart, V.J.; Lineen, E.B.; Badiye, A.; Byers, P.M.; Patel, A.; Vianna, R.; Koerner, M.M.; El Banayosy, A.; Loebe, M.; et al. A Weaning Protocol for Venovenous Extracorporeal Membrane Oxygenation with a Review of the Literature. Artif. Organs 2018, 42, 605-610. [CrossRef]

49. Vasques, F.; Romitti, F.; Gattinoni, L.; Camporota, L. How I wean patients from veno-venous extra-corporeal membrane oxygenation. Crit. Care 2019, 23, 1-3. [CrossRef]

50. Costa, E.L.V.; Amato, M.B.P. Ultra-protective tidal volume: How low should we go? Crit. Care 2013, 17, 1-2. [CrossRef]

51. Umbrello, M.; Marino, A.; Chiumello, D. Tidal volume in acute respiratory distress syndrome: How best to select it. Ann. Transl. Med. 2017, 5, 287. [CrossRef]

52. Santos, R.S.; Maia, L.D.A.; Oliveira, M.V.; Santos, C.L.; Moraes, L.; Pinto, E.F.; Samary, C.D.S.; Machado, J.A.; Carvalho, A.C.; Fernandes, M.V.D.S.; et al. Biologic Impact of Mechanical Power at High and Low Tidal Volumes in Experimental Mild Acute Respiratory Distress Syndrome. Anesthesiol. 2018, 128, 1193-1206. [CrossRef]

53. Araos, J.; Alegria, L.; Garcia, P.; Cruces, P.; Soto, D.; Erranz, B.; Amthauer, M.; Salomon, T.; Medina, T.; Rodriguez, F.; et al. Near-Apneic Ventilation Decreases Lung Injury and Fibroproliferation in an Acute Respiratory Distress Syndrome Model with Extracorporeal Membrane Oxygenation. Am. J. Respir. Crit. Care Med. 2019, 199, 603-612. [CrossRef]

54. Peek, G.J.; Clemens, F.; Elbourne, D.; Firmin, R.; Hardy, P.; Hibbert, C.; Killer, H.; Mugford, M.; Thalanany, M.; Tiruvoipati, R.; et al. CESAR: Conventional ventilatory support vs extracorporeal membrane oxygenation for severe adult respiratory failure. BMC Heal. Serv. Res. 2006, 6, 163. [CrossRef] [PubMed]

55. Patroniti, N.; Bonatti, G.; Senussi, T.; Robba, C. Mechanical ventilation and respiratory monitoring during extracorporeal membrane oxygenation for respiratory support. Ann. Transl. Med. 2018, 6, 386. [CrossRef] [PubMed]

56. Marini, J.J.; Rocco, P.R. Which component of mechanical power is most important in causing VILI? Crit. Care 2020, $24,1-3$. [CrossRef] [PubMed] 